$\$$ Research Square

\title{
Prevalence of Anosmia and Ageusia in Patients with COVID-19 at a Primary Health Center, Doha, Qatar
}

\author{
Raid Muhmid Al-Ani ( $\nabla$ raedalani2003@yahoo.com ) \\ University of Anbar, College of Medicine, Department of Surgery/ENT. \\ DEBASHIS ACHARYA \\ Primary Health Care Corporation (P.H.C.C.), Govt. of QATAR
}

\section{Research Article}

Keywords: Anosmia, Ageusia, Prevalence, COVID-19, Qatar

Posted Date: July 29th, 2020

DOI: https://doi.org/10.21203/rs.3.rs-48737/v1

License: (9) This work is licensed under a Creative Commons Attribution 4.0 International License. Read Full License

Version of Record: A version of this preprint was published at Scholarly Journal of Otolaryngology on August 4th, 2020. See the published version at https://doi.org/10.32474/SJ0.2020.04.000197. 


\section{Abstract}

Background: Loss of smell and taste are common complaints in patients with the COVID-19 disease. These symptoms may present alone or with other symptoms. It is of utmost importance to know their rates of occurrence for better controlling of the infection.

Objectives: To detect the prevalence of anosmia and ageusia in individuals with COVID-19 in Al-Wajbah Primary Health Center, Doha, Qatar.

Materials and methods: This retrospective study was conducted at Al-Wajbah Primary Health Center, Doha, Qatar. The study covered the two-month period -May and June 2020. The proven cases of COVID19 by real-time PCR (Polymerase Chain Reaction) were enrolled in the study. Data regarding the age, gender, symptomatology including anosmia and ageusia, history of recent travel, smoking, past history of nasal and paranasal diseases (NPND), and severity of the disease were taken from the patients' records. IBM-SPSS version 22 statistical software was used for the analysis of the data.

Results: Out of $141,35(24.82 \%)$ subject presented with anosmia, ageusia or both. Most of the patients were from age group $>30$ year $(n=104,73.76 \%)$ with nearly equal gender. The majority of the individuals were without history of recent travel (92.2\%) and smoking (80.14\%). Three-quarters of the patients were asymptomatic, and $51.06 \%$ with a past history of NPND. The male sex, history of recent travel, smoking, and severe course of the disease were positive, highly significant association with anosmia or ageusia. All patients returned to their normal smell and taste sensations within a mean duration of 6.89 days.

Conclusion: Loss of taste and smell were common symptomatology of COVID-19 disease. The males, recent travel, smoking, and severe course of the disease were risk factors of the anosmia and ageusia in COVID-19 cases.

\section{Introduction}

On the last day of the year 2019, 41 patients with pneumonia of unidentified cause were detected in Wuhan City, Hubei province in China [1]. The identification of the causative microorganism was reached from the examination of the throat swab samples from the Chinese Centre for Disease Control and Prevention (CCDC) on $7^{\text {th }}$ January, 2020. The causative agent was named Severe Acute Respiratory Syndrome Coronavirus 2 (SARS-CoV-2). The World Health Organization (WHO) in February 2020 named the disease as COVID-19 [2]. During the outbreak of COVID-19 infection, there were millions of infected people and hundreds of thousands dead all over the world, and it remains a global threat [3].

The attention worldwide focused mainly on the infected cases and those with direct contact with patients. The asymptomatic carriers were not being tested because of the shortage in testing kits including all the suspicious cases even in developed countries with a good health system like the US and 
the UK [4]. So, it is of utmost importance to evaluate the number of predictive symptoms of this disease in order to make a decision for a self-isolation and prevent the spreading of the infection.

At the initial times of COVID-19 disease, the symptoms of the inpatient cases in China included fatigue, fever, dry and productive cough, shortness of breath, chest compression, myalgia, diarrhea, vomiting, anorexia, headache, sore throat, dizziness, palpitations, and, chest pain [5] [6].

After that, there have been an increment in the scientific studies from various parts of the world about the significant increase in the cases number presenting with loss of smell alone. Gilani et al. from Iran reported 8 patients with anosmia, 5 of them were confirmed cases of COVID-19, and the remaining 3 cases were not tested owing to the shortage of testing kits in the pandemic period [7]. An European multicenter study concluded that olfactory (85.6\%) and gustatory (88\%) abnormalities are prevalent symptoms in European confirmed COVID-19 cases, who may not have other nasal complaints [8]. However, these cases do not meet the criteria of self-isolation or testing [9].

Anosmia and/or ageusia might present alone or in mild confirmed cases of COVID-19. It is therefore necessary to test or quarantine those individuals with these complaints.

We aimed to determine the prevalence of anosmia and ageusia in patients with COVID-19 in Al-Wajbah Primary Health Center, Doha, Qatar.

\section{Materials And Methods}

This retrospective study was conducted at Al-Wajbah Primary Health Center, Doha, Qatar during May and June 2020. Patients with proven COVID-19 infection by real-time polymerase chain reaction (PCR) on nasopharyngeal and oropharyngeal swabs were enrolled in the current study. The PCR swabs were tested at the pathological laboratory of Hamad Medical Corporation, Qatar for the corona virus using the Abbot m2000 Real Time device patented in South Africa. The study was approved by the Ethical Committee of the University of Anbar (reference number 34 on 28-7-2020) and verbal consent was taken from every patient or their parents to publish their data in a scientific article.

Subjects with incomplete data, previous anosmia or ageusia, psychological disturbances, and those who lost to follow-up (not responding to 3 telephone calls) were excluded from the study. We had 180 COVID19 positive patients during our study period, out of which only 141 patients were included in this study.

ICleanhcy Specimen Collection Flocked nylon swabs-Huachenyang (Hcy) made in USA was used to collect the samples from all the individuals.

Data regarding the age, gender, history of recent travel, presenting symptoms (fever, myalgia, cough, dyspnea, fatigability, sore throat, anosmia, ageusia, diarrhea, etc.), past nasal and paranasal diseases (NPND), history of smoking, and the severity of the disease were taken from every patient who tested positive. 
The patients were divided into asymptomatic and symptomatic. The severity of the symptomatic diseases were classified into [10]:

1. Mild patients complained from only low-grade fever, mild cough, and slight fatigue.

2. Moderate patients complained from high grade fever and moderate respiratory symptoms. Findings of pneumonitis were seen in chest radiograph.

3. Severe patients had dyspnea, respiratory rate $30 / \mathrm{min}$, blood oxygen saturation $93 \%$, partial pressure of arterial oxygen to fraction of inspired oxygen ratio $300 \mathrm{~mm} \mathrm{Hg}$, or CT scans showing at least a $50 \%$ increase in infiltrate volume over $24-48$ hours.

4. Critical patients had respiratory failure, septic shock, and/or multiple-organ dysfunction or failure.

IBM-SPSS statistical software version 22 was used for analyzing the data. Chi-Square test was used to compare between categorially variables. Independent-Samples T-test was used for the comparison of the means. P-value was considered a statistically significant difference if it is less than 0.05 .

\section{Results}

Out of 180, 141 patients fulfilled the inclusion and exclusion criteria, were thus enrolled in the study. The prevalence rates of ageusia and anosmia, ageusia, and anosmia were $8.51 \%, 11.35 \%$, and 4.96 respectively as shown in Table 1.

Table 1: The distribution of 141 patients with COVID-19 disease.

\begin{tabular}{|l|l|l|l|}
\hline \multicolumn{2}{|c|}{ COVID- 19 patients } & Frequency & Percentage \\
\hline \multirow{4}{*}{ With ageusia or anosmia or both } & Ageusia and anosmia & 12 & 8.51 \\
\cline { 2 - 4 } & Ageusia & 16 & 11.35 \\
\cline { 2 - 4 } & Anosmia & 7 & 4.96 \\
\cline { 2 - 4 } & Total & 35 & 24.82 \\
\hline Without ageusia and anosmia & & 106 & 75.18 \\
\hline Total & \multicolumn{2}{|c|}{141} & 100 \\
\hline
\end{tabular}

The age of the patients ranged from 3-56 years with a mean age of 35.91year \pm 10.069 . The age range of the patients without anosmia 3-56 years was wider than those with anosmia 26-53 years, and there was a high statistically significant difference between the mean ages of both groups ( $p$-value=0.000) Table 2 . The highest age group affected by COVID-19 disease was $>30$ years $(n=104,73.76 \%)$. The males 71 (50.35\%) was slightly more than females $70(49.65 \%)$. Most of our patients were without history of recent travel $130(92.2 \%)$. The majority of the cases were without history of smoking $(80.14 \%)$. Three-quarters of the cases were asymptomatic as shown in Tables $\mathbf{2}$ and $\mathbf{3}$. There was a positively statistically significant difference between the 2 groups of patients (with and without anosmia) regarding the following factors the male sex, history of recent travel, smokers, and severe course of the disease. While, there was no statistically significant difference between both groups regarding the age group and past history of NPND ( $p$-value $>0.05$ ) as shown in Table 2. The age ranged of the subjects without ageusia 3-56 years was wider 
than those with ageusia 10-53 years, and there was a high statistically significant difference between the mean ages of both groups ( $p$-value $=0.000$ ) Table 3 . There was a positively statistically significant difference between the 2 groups of patients (with and without ageusia) regarding the following factors the male sex, history of recent travel, smokers, past history of NPND, and severe course of the disease. While, there was no statistically significant difference between both groups regarding the age groups of the patients ( $p$-value>0.05) as shown in Table 3. All patients were showed full recovery from the anosmia and ageusia with a resolution time of 3-12 days with a mean of 6.89 days \pm 3.056 . No death was reported in our study.

Table 2: The relationship between socio-clinical characteristics and anosmia of 141 patients with COVID-19.

\begin{tabular}{|c|c|c|c|c|}
\hline Variables & $\begin{array}{l}\text { With anosmia } \\
\text { Number/\% }\end{array}$ & $\begin{array}{l}\text { Without anosmia } \\
\text { Number } / \%\end{array}$ & $\begin{array}{l}\text { Total } \\
\text { Number/\% }\end{array}$ & p-value \\
\hline Mean age & $\begin{array}{l}26-53 \text { years } \\
37.16 \pm 8.546\end{array}$ & $\begin{array}{l}3-56 \text { years } \\
35.71 \pm 10.303\end{array}$ & & 0.000 \\
\hline \multicolumn{4}{|l|}{ Age groups } & \multirow{4}{*}{0.259} \\
\hline$\leq 30$ years & 7 (18.9) & $30(81.1)$ & $37(26.24)$ & \\
\hline$>30$ years & $12(11.5)$ & $92(88.5)$ & $104(73.76)$ & \\
\hline Total & 19 (13.5) & $122(86.5)$ & $141(100)$ & \\
\hline \multicolumn{4}{|l|}{ Gender } & \multirow{4}{*}{0.029} \\
\hline Male & $14(19.7)$ & $57(80.3)$ & $71(50.35)$ & \\
\hline Female & $5(7.1)$ & $65(92.9)$ & $70(49.65)$ & \\
\hline Total & $19(13.5)$ & $122(86.5)$ & $141(100)$ & \\
\hline \multicolumn{4}{|l|}{ Recent travel } & \multirow{4}{*}{0.001} \\
\hline Yes & $5(45.5)$ & $6(54.5)$ & $11(7.8)$ & \\
\hline No & $14(10.8)$ & $116(89.2)$ & $130(92.2)$ & \\
\hline Total & $19(13.5)$ & $122(86.5)$ & $141(100)$ & \\
\hline \multicolumn{4}{|l|}{ Smoking } & \multirow{4}{*}{0.000} \\
\hline Yes & $13(46.4)$ & $15(53.6)$ & $28(19.86)$ & \\
\hline No & $6(5.3)$ & $107(94.7)$ & $113(80.14)$ & \\
\hline Total & $19(13.5)$ & $122(86.5)$ & $141(100)$ & \\
\hline \multicolumn{4}{|c|}{ Past history of NPND } & \multirow{4}{*}{0.104} \\
\hline Yes & $13(18.1)$ & 59 (81.9) & $72(51.06)$ & \\
\hline No & $6(8.7)$ & $63(91.3)$ & $69(48.94)$ & \\
\hline Total & 19 (13.5) & $122(86.5)$ & $141(100)$ & \\
\hline \multicolumn{4}{|l|}{ Severity } & \multirow{6}{*}{0.000} \\
\hline Asymptomatic & $0(0)$ & 107 (100) & $106(75.17)$ & \\
\hline Mild & $9(42.9)$ & $12(57.1)$ & $22(15.61)$ & \\
\hline Moderate & $6(75)$ & $2(25)$ & 8 (5.67) & \\
\hline Severe & $4(80)$ & $1(20)$ & $5(3.55)$ & \\
\hline Total & 19 (13.5) & $122(86.5)$ & $141(100)$ & \\
\hline
\end{tabular}

Table 3: The relationship between socio-clinical characteristics and ageusia of 141 patients with COVID-19. 


\begin{tabular}{|c|c|c|c|c|}
\hline Variables & $\begin{array}{l}\text { With ageusia } \\
\text { Number/\% }\end{array}$ & $\begin{array}{l}\text { Without ageusia } \\
\text { Number/\% }\end{array}$ & $\begin{array}{l}\text { Total } \\
\text { Number/\% }\end{array}$ & p-value \\
\hline Mean age & $\begin{array}{l}10-53 \text { years } \\
37.29 \pm 10.029\end{array}$ & $\begin{array}{l}3-56 \text { years } \\
35.57 \pm 10.094\end{array}$ & & 0.000 \\
\hline \multicolumn{4}{|l|}{ Age groups } & \multirow[t]{4}{*}{0.868} \\
\hline$\leq 30$ years & 7 (18.9) & $30(81.1)$ & $37(26.24)$ & \\
\hline$>30$ years & $21(20.2)$ & $83(79.8)$ & $104(73.76)$ & \\
\hline Total & $28(19.9)$ & $113(80.1)$ & $141(100)$ & \\
\hline \multicolumn{4}{|l|}{ Gender } & \multirow{4}{*}{0.013} \\
\hline Male & $20(28.2)$ & $51(71.8)$ & $71(50.35)$ & \\
\hline Female & $8(11.4)$ & $62(88.6)$ & $70(49.65)$ & \\
\hline Total & 28 (19.9) & $113(80.1)$ & $141(100)$ & \\
\hline \multicolumn{4}{|l|}{ Recent travel } & \multirow{4}{*}{0.000} \\
\hline Yes & 7 (63.6) & $4(36.4)$ & $11(7.8)$ & \\
\hline No & $21(16.2)$ & 109 (83.8) & $130(92.2)$ & \\
\hline Total & $28(19.9)$ & $113(80.1)$ & $141(100)$ & \\
\hline \multicolumn{4}{|l|}{ Smoking } & \multirow{4}{*}{0.000} \\
\hline Yes & $18(64.3)$ & $10(65.7)$ & $28(19.86)$ & \\
\hline No & $10(8.8)$ & $103(91.2)$ & $113(80.14)$ & \\
\hline Total & 28 (19.9) & $113(80.1)$ & $141(100)$ & \\
\hline \multicolumn{4}{|c|}{ Past history of NPND } & \multirow{4}{*}{0.000} \\
\hline Yes & $23(31.9)$ & $49(68.1)$ & $72(51.06)$ & \\
\hline No & $5(7.2)$ & $64(92.8)$ & $69(48.94)$ & \\
\hline Total & 28 (19.9) & $113(80.1)$ & $141(100)$ & \\
\hline \multicolumn{4}{|l|}{ Severity } & \multirow{6}{*}{0.000} \\
\hline Asymptomatic & $0(0)$ & $106(98.2)$ & $106(75.17)$ & \\
\hline Mild & $15(68.2)$ & 7 (31.8) & $22(15.61)$ & \\
\hline Moderate & $8(100)$ & $0(0)$ & $8(5.67)$ & \\
\hline Severe & $5(100)$ & $0(20)$ & 5 (3.55) & \\
\hline Total & $28(19.9)$ & $113(80.1)$ & $141(100)$ & \\
\hline
\end{tabular}

\section{Discussion}

Chemosensory dysfunctions are common problems during the pandemic COVID-19 crisis. Smell and taste Abnormalities (STA) were the first and the only complaints in $10 \%$ of subjects, $19 \%$ experienced STA before other classical symptoms like fever and cough, as well as $25 \%$ of the children had only STA at the time of the presentation [10]. Therefore, these disorders are indicators of early cases of COVID-19 disease and necessary for screening and infection control. Our study design included a questionnaire for patients about anosmia, ageusia, or both only to overcome the bias due to unrecognized pre-existing chemosensory hypo-function, and the difficulties in catching these hypo-functions as they are highly subjected to personal variation in response to the questioner, and the stressful conditions of the patients. The prevalence rates of ageusia and anosmia, ageusia, and anosmia were $8.51 \%, 11.35 \%$, and $4.96 \%$, respectively, making the overall prevalence rate $24.82 \%$ Table 1 . Qiu et al. multi-centric case series study found that $41 \%$ of the COVID-19 patients complained from STA [10]. Speth et al. reported that the prevalence rate of olfactory dysfunction was $61.2 \%$ [11]. Paderno et al. showed that the olfactory and gustatory dysfunctions were seen in $83 \%$ and $89 \%$ of patients, respectively [12]. In a systematic review 
and meta-analysis study by Tong et al. found that the prevalence rate of the 10 investigations which analyzed the olfactory dysfunction in patients with COVID-19 disease was $52.73 \%$. While nine investigations reported that the prevalence rate of the gustatory dysfunction was $43.93 \%$ [13]. The study by Chary et al. found that the rate of STA without nasal complaints was 70\% [14]. The variability among various studies might be attributed to the variation in the ethnicity, sample size, and type of the sample (children or adults or both, hospitalized, non-hospitalized or both, with or without nasal symptoms, subjective, objective or both assessment, and severity of the disease).

There are no proved mechanisms for the STA. There is no prior investigation that studied the possible association between coronavirus and the appearance of taste alteration [15]. The study by Zhou et al. have been confirmed that COVID-19 uses the same receptor [cellular angiotensin-converting enzyme 2 (ACE 2)] as SARS-CoV [16]. The enzyme found in the mouth particularly on the tongue. Therefore, it is possible that the COVID-19 causes taste dysfunction same as the ACE2 inhibitors [17]. Secondly, the taste disturbance in the presence of smell abnormality is that both chemosensory senses are intimately correlated [18]. The study by Brann et al. [19] suggested that the COVID-19 virus affects the non-neuronal olfactory epithelium causing anosmia and the associated taste dysfunctions. This is the most acceptable mechanism because most of the patients with COVID-19 infection are of the mild severity, outpatient cases, and most of the STA resolve within short period [8][20]. However, a large number of a cohort investigation with long term follow-up and objective measures of the assessment are needed to assess the exact time and rate of recovery of the STA in confirmed cases of COVID-19 infection.

In a study by Vaira et al. [21] found that $66 \%$ of patients reported spontaneous resolution of STA on subjective self-reported questionnaire. While, $80 \%$ of these individuals were still revealed a slight disturbance in their chemosensory functions on objective evaluation measures. The authors revealed two possible causes for this observation. Firstly, the patients might be suffering from preexisting unnoticed chemosensory dysfunctions and secondly, the patients might consider the great improvement in these senses as normal. The present study was used the self-reported questionnaire on the evaluation of the anosmia or ageusia resolution in COVID-19 cases. Fortunately, all patients showed resolution with a mean of 6.89 days. This finding was higher than what was reported in the prior investigations [8][21]. However, self-reported evaluation was a shortcoming of the present study. we recommend a further study to use objective assessment of these chemosensory alterations in COVID-19 cases.

The study found that there was an equal gender distribution, but males with anosmia and ageusia were more prevalent than females ( $p$-value $<0.05$ ). This finding was in contrast to the prior studies [8][10][11] [12][14][22][21]. This difference between our study and the previous studies might be attributed to the differences in the social and cultural behaviors and geographical locations.

Many investigations reported that younger patients had a positive impact on the resolution of the STA [11][14][21]. Despite, approximately $75 \%$ of the patients were more than 30 years, our study found that the age was not considered a significant factor in relation with a resolution of anosmia and ageusia ( $p$ value $>0.05)$. Similar finding was reported by the previous study [12]. 
In the early period of COVID-19 disease, recent travel is considered as an important factor for the transmission of the disease. But as the time progresses and the infection becomes a worldwide disease, recent travel becomes less important. Moreover, the contact with suspicious or proved COVID-19 cases is an important way of the transmission of the infection to healthy people. Our findings revealed that recent travel had a significant factor for those patients with anosmia, ageusia or both ( $p$-value $<0.05)$. However, the study didn't find a cause for this difference.

Smoking has adverse effects on the health state of the lungs and the human immune system. Therefore, smokers are more vulnerable to acquire infectious conditions. Previous investigations showed that the smokers are twice more infected with influenza than non-smokers as well as they are associated with a more severe course of illness. Besides, there were more deaths among smokers than the non-smoker during the MERS-CoV outbreak [23]. Moreover, smoking has a negative impact factor on the severity and clinical outcome in patients with the COVID-19 disease [23]. However, our study reported low prevalence of smokers (20\%) in patients with the COVID-19 disease. This finding was similar to Speth et al. study [11] who they reported that $8.8 \%$ of their patients were current smokers. The current study and Speth et al. studies didn't find an explanation of this contradiction. Despite, the low prevalence of smokers in the present study, the smoking was a highly statistically significant association with anosmia and ageusia (p-value=0.000).

It is obvious that the nasal and paranasal problems like nasal polyposis have a drawback on the smell because they interfere with air current from reaching the olfactory epithelium in the roof of the nose. Besides, they might give some sort of taste hypo-function. The study by Paderno et al. [12] reported that nasal congestion is a risk factor for late recovery of the STA in the COVID-19 cases. Speth et al. [11] found that patients with this disease had also allergic rhinitis (35\%), chronic rhinosinusitis (1\%), and asthma (12.6\%). Although, half of our cases gave NPNDs, but they weren't considering a significant association with anosmia ( $p$-value $=0.104$ ). The highly significant association between the NPNDs and ageusia was found in our study. Therefore, it is logical to ask in the history about these problems when one wants to manage a case of the STA.

Olfactory dysfunction is generally a first symptom in COVID-19 disease [8] [11][23]. The study by Yan et al. [24] reported that the mild disease had a statistically significant association with the STA. Severe disease type is usually not associated with the STA. Therefore, the STA can act as a major positive factor for the prognosis of COVID-19 disease. However, this theory is of limited in actual clinical practice because the hospitalized patients in the critical care unit aren't able to give interview due to their bad health condition [14]. Fortunately, there was no patient in our study needed admission to the intensive care unit. The current study found a statistically significant association between anosmia and ageusia and severe disease type. This finding was in contrast with Yan et al.[24]. This may be attributed to the difference in the inclusion criteria (anosmia and ageusia in our study versus STA in Yan et al's study.)

The limitations of the study included small sample size, didn't register the time of onset of anosmia and ageusia, and the study was not used for objective measures of assessment. 
In conclusion, the prevalence of anosmia and ageusia was $24.82 \%$. They occur mostly in the age group $>30$ years with equal sex distribution. Most patients without history of smoking and recent travel. Half of the patients gave a past history of NPNDs. The majority of the cases were asymptomatic. The male sex, recent travel, smoking, severe type of the disease were considered risk factors of the anosmia and ageusia. Resolution of these symptoms occurred in $100 \%$ of the involved patients with a mean resolution time of 6.89 days.

\section{Declarations}

\section{Conflicts of interest}

The authors declare that they have no competing interests.

\section{References}

[1] H. Lu, C. W. Stratton, and Y. Tang, "Outbreak of Pneumonia of Unknown Etiology in Wuhan China: the Mystery and the Miracle," J. Med. Virol.

[2] W. H. Organization, “WHO Director-General's remarks at the media briefing on 2019-nCoV on 11 February 2020," Internet] World Heal. Organ., 2020.

[3] E. Mahase, "Covid-19: WHO declares pandemic because of 'alarming levels' of spread, severity, and inaction." British Medical Journal Publishing Group, 2020.

[4] C. Menni et al., "Loss of smell and taste in combination with other symptoms is a strong predictor of COVID-19 infection," medRxiv, 2020.

[5] Z. Wu and J. M. McGoogan, "Characteristics of and important lessons from the coronavirus disease 2019 (COVID-19) outbreak in China: summary of a report of 72314 cases from the Chinese Center for Disease Control and Prevention," Jama, vol. 323, no. 13, pp. 1239-1242, 2020.

[6] Z. Wang, B. Yang, Q. Li, L. Wen, and R. Zhang, "Clinical features of 69 cases with coronavirus disease 2019 in Wuhan, China," Clin. Infect. Dis., 2020.

[7] S. Gilani, R. Roditi, and M. Naraghi, "COVID-19 and Anosmia in Tehran, Iran," Med. Hypotheses, p. 109757, 2020.

[8] J. R. Lechien et al., "Olfactory and gustatory dysfunctions as a clinical presentation of mild-tomoderate forms of the coronavirus disease (COVID-19): a multicenter European study," Eur. Arch. OtoRhino-Laryngology, pp. 1-11, 2020.

[9] C. Hopkins and N. Kumar, "Loss of sense of smell as marker of COVID-19 infection," ENT UK [https//Www. entuk. org/sites/default/files/files/Loss sense smell as marker COVID. pdf] Date accessed, 
vol. 26 , no. 03, p. 2020, 2020.

[10] C. Qiu et al., "Olfactory and Gustatory Dysfunction as An Early Identifier of COVID-19 in Adults and Children: An International Multicenter Study," medRxiv, 2020.

[11] M. M. Speth, T. Singer-Cornelius, M. Obere, I. Gengler, S. J. Brockmeier, and A. R. Sedaghat, "Olfactory Dysfunction and Sinonasal Symptomatology in COVID-19: Prevalence, Severity, Timing, and Associated Characteristics," Otolaryngol. Neck Surg., 2020.

[12] A. Paderno et al., "Olfactory and Gustatory Outcomes in COVID-19: A Prospective Evaluation in Nonhospitalized Subjects," Otolaryngol. Neck Surg.

[13] J. Y. Tong, A. Wong, D. Zhu, J. H. Fastenberg, and T. Tham, "The prevalence of olfactory and gustatory dysfunction in COVID-19 patients: a systematic review and meta-analysis," Otolaryngol. Neck Surg., p. $0194599820926473,2020$.

[14] E. Chary et al., "Prevalence and Recovery From Olfactory and Gustatory Dysfunctions in Covid-19 Infection: A Prospective Multicenter Study," Am. J. Rhinol. Allergy, p. 1945892420930954, 2020.

[15] R. M. Al-Ani, "Smell and Taste Abnormalities due to COVID-19," Al-Anbar Med. J., vol. 16, no. 1, pp. 1$2,2020$.

[16] P. Zhou et al., "A pneumonia outbreak associated with a new coronavirus of probable bat origin," Nature, vol. 579, no. 7798, pp. 270-273, 2020.

[17] J. Suliburska, G. Duda, and D. Pupek-Musialik, "The influence of hypotensive drugs on the taste sensitivity in patients with primary hypertension," Acta Pol Pharm, vol. 69, no. 1, pp. 121-127, 2012.

[18] D. M. Small and J. Prescott, "Odor/taste integration and the perception of flavor," Exp. brain Res., vol. 166, no. 3-4, pp. 345-357, 2005.

[19] D. Brann, T. Tsukahara, C. Weinreb, D. W. Logan, and S. R. Datta, "Non-neural expression of SARSCoV-2 entry genes in the olfactory epithelium suggests mechanisms underlying anosmia in COVID-19 patients," bioRxiv, 2020.

[20] C. H. Yan, F. Faraji, D. P. Prajapati, C. E. Boone, and A. S. DeConde, "Association of chemosensory dysfunction and Covid-19 in patients presenting with influenza-like symptoms," in International Forum of Allergy \& Rhinology, 2020.

[21] L. A. Vaira et al., "Objective evaluation of anosmia and ageusia in COVID-19 patients: Single-center experience on 72 cases," Head Neck, vol. 42, no. 6, pp. 1252-1258, 2020.

[22] Y. Lee, P. Min, S. Lee, and S.-W. Kim, "Prevalence and duration of acute loss of smell or taste in COVID-19 patients," J. Korean Med. Sci., vol. 35, no. 18, 2020. 
[23] R. Kaye, C. W. D. Chang, K. Kazahaya, J. Brereton, and J. C. Denneny III, "COVID-19 anosmia reporting tool: initial findings," Otolaryngol. Neck Surg., p. 0194599820922992, 2020.

[24] C. H. Yan, F. Faraji, D. P. Prajapati, C. E. Boone, and A. S. DeConde, "Association of chemosensory dysfunction and Covid-19 in patients presenting with influenza-like symptoms [published online April 12, 2020]," in Int Forum Allergy Rhinol. 